\title{
Interpreting the ambulatory glucose profile
}

\author{
PETER HAMMOND
}

\begin{abstract}
A systematic approach to interpreting dense glucose data from continuous glucose monitoring (CGM) systems is important for dealing with the daunting amount of data that these systems provide. Moreover, a consensus approach would be useful to reduce differences in interpretation of similar data between physicians. The ambulatory glucose profile (AGP), now available for the FreeStyle Libre flash glucose monitoring system and some CGM systems, allows simpler and more confident identification of patterns, by averaging data for the modal day (itself derived from several daily CGM traces). Its output provides a single curve representing the average, with interquartile and interdecile ranges. Even scattered individual data values without discernible pattern can be averaged and produce useful clinically relevant information, especially where there is considerable glycaemic variability. Consensus recommendations on the use of the AGP build on an understanding of the user's daily routine, followed by examination of the AGP for periods of hypoglycaemic risk, i.e. when the 10th centile line for glucose approaches or enters the hypoglcycaemic range. These analyses are supported by the glucose pattern insights analysis available from the FreeStyle Libre flash glucose monitoring system, which flags up the likelihood of low glucose, the proximity of the median glucose to target, and the degree of variability below the median at various times of day. Where hypoglycaemia patterns are not apparent, the consensus guidance recommends assessing the periods around overnight, morning meal, midday meal, and evening meal in turn. In these ways, the consensus guideline provides the systematic approach to interpretation which is the cornerstone of using the AGP.

Br J Diabetes 2016;16(Suppl1):S10-S15
\end{abstract}

Key words: continuous glucose monitoring, flash glucose monitoring, ambulatory glucose profile, blood glucose variability, hypoglycaemia

\section{Harrogate and District NHS Foundation Trust, UK}

Address for correspondence: Peter Hammond Diabetes Resource Centre, Harrogate District Hospital, Lancaster Park Road, Harrogate, HG2 7SX, UK

E-mail: peterhammond@nhs.net

http://dx.doi.org/10.15277/bjd.2016.072
Challenges in interpreting dense glucose data

A systematic approach to interpreting dense glucose data such as continuous glucose monitoring (CGM) data is important for any healthcare professional who has to decipher the salient clinical information from what can be a daunting amount of data. Moreover, a consensus approach to this will mean that all clinicians should reach fairly similar conclusions to each other when looking at the same data.

We have generally advocated that the approach to analysis should be based on the following sequence: modal day; individual daily profiles; then breaking the glucose traces into overnight, fasting/pre-meal and post-meal phases; and finally looking at the impact of other factors such as exercise, alcohol and work patterns. In this way the user can be educated to make changes to both their insulin regimen and lifestyle to optimise glycaemic control. ${ }^{1}$

The modal day superimposes traces from each day, a sensor daily overlay, to try and identify trends which occur on a regular basis. However in many cases the degree of variation from day to day means that no pattern is easily identified. Furthermore the more days' data that are superimposed the more likely it is that any pattern that may be present will be obscured (Figure 1). Traditional CGM software does give an average glucose curve but it is difficult to be sure of the reliability of this in the face of apparently highly variable glucose levels.

\section{Introducing the ambulatory glucose profile}

The ambulatory glucose profile (AGP), which is now available for the FreeStyle Libre flash glucose monitoring system and some CGM systems, allows simpler and more confident identification of patterns. As we have already seen, the AGP averages the data used to create the modal day and provides a single curve representing the average, with interquartile and interdecile ranges shaded to demonstrate the glycaemic variability. Figure 2 shows how scattered individual data values without discernible pattern can be averaged and produce useful clinically relevant information.

There are occasions when a review of downloaded dense glucose data produces little information on which to base therapeutic decisions. AGP may provide the additional analysis in such cases to allow useful adjustments to be made. Where there is little glycaemic variability, as is often the case overnight, the 25th-75th centile lines and 10th-90th centile will be close to the median and this provides confidence that the trends observed are consistent over time. However this is usually apparent from the raw data of the sensor overlay. It is when there is con- 
Figure 1. Superimposed continuous glucose monitoring traces from a single patient highlight the difficulty in identifying patterns that will support optimisation of therapy

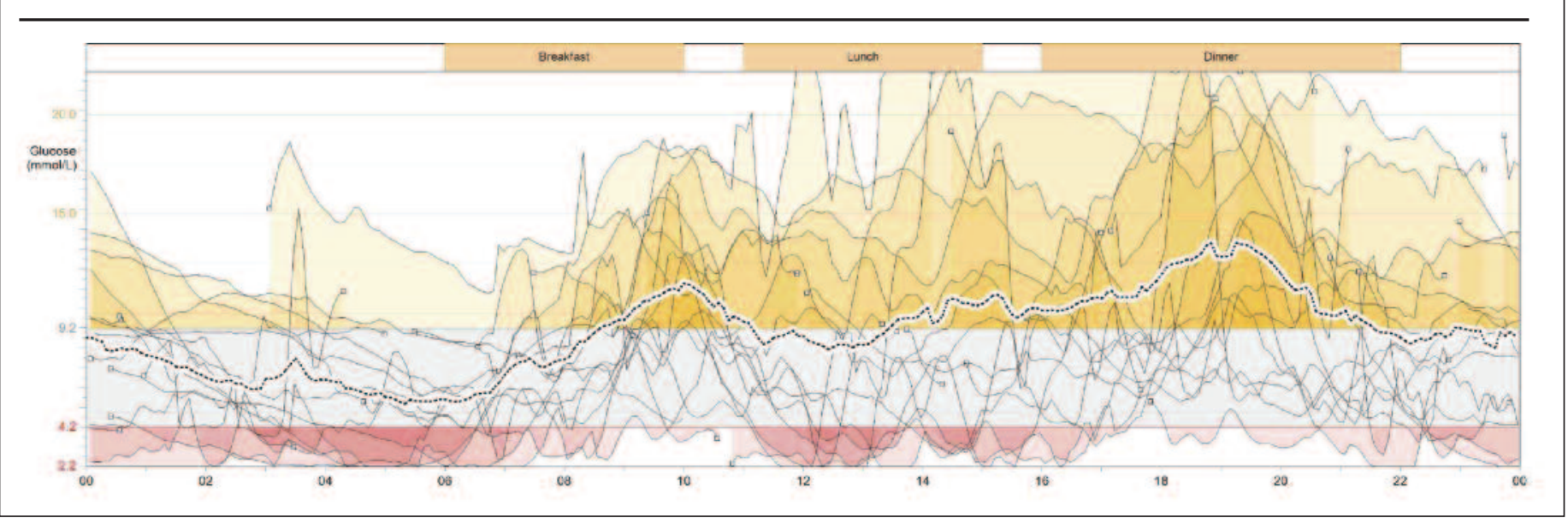

Figure 2. Scattered individual data values without discernible pattern can be averaged to produce useful clinically relevant information

Daily Patterns (with glucose readings)
17 July $2015-16$ October 2015 (92 days)

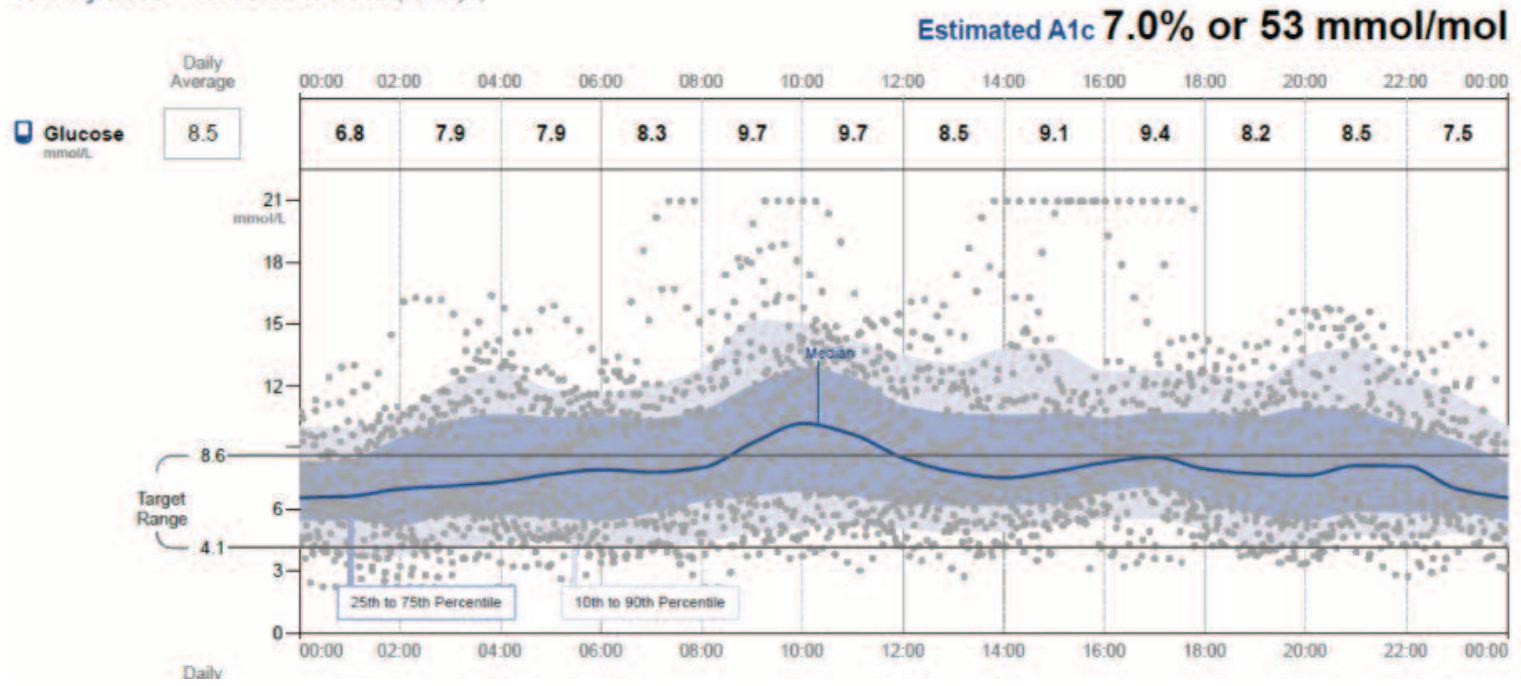

siderable glycaemic variability that AGP can provide more certainty with regard to interpreting trends over time. Often there will be more variation to one side of the median which gives more confidence when identifying hyperglycaemic or hypoglycaemic trends, and the potential consequences of treatment change are more readily identified. For example, an increase in insulin dose to address a high median glucose level during a certain period of the day might significantly increase the risk of hypoglycaemia if there is a lot of variability below the mean, but not if there is little variability below the mean.

Whenever assessing such data it is useful to have as much information as possible about what the user has been doing, particularly insulin dosing, bolus ratios, physical activity and alcohol intake, but also any other external factors which the user thinks may influence their blood glucose levels. This is equally true when it comes to interpreting AGP data, although in more general terms e.g. what time are meals generally taken; is there a particular time of day when exercise is typically taken.

\section{Consensus recommendations on using the AGP}

Consensus recommendations for use of AGP (Figure 3$)^{2}$ advocate a review of the data quality, followed by understanding the user's typical daily routine. The next step should be to assess for hypoglycaemia patterns. Figure 4 shows data over 92 days from a patient using continuous subcutaneous insulin infusion, with an average total daily dose of $43.1 \pm 3.8$ units, $58 \%$ bolus. When 
Figure 3. Consensus recommendations for the use of ambulatory glucose profiling $(A G P)^{2}$

To improve glycaemic control, it is highly recommended that the data are reviewed with the patient in order to discuss areas of concern

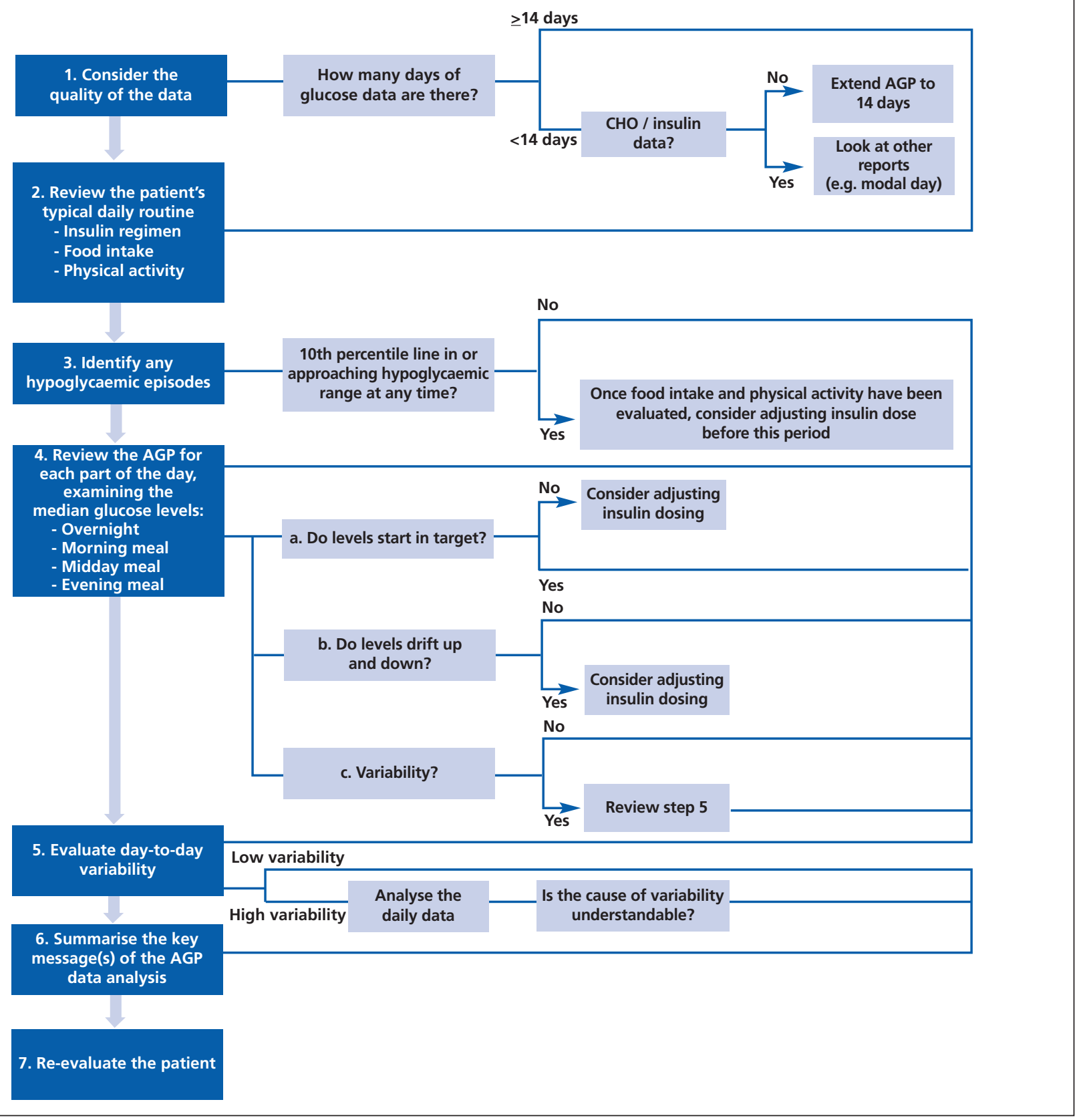

looking for hypoglycaemia patterns, the aim is to identify times of day when the 10th centile line approaches or enters the hypoglcycaemic range. The raw scattered data (Figure 4a) shows episodes of biochemical hypoglycaemia occurring throughout the day. When the AGP is viewed in isolation (Figure $4 b$ ) it becomes clear that hypoglycaemia risk is greatest between 00.00 and 08.00, with a short period of increased risk between 18.30 and 20.30 .

The consensus guideline prompts that, if hypoglycaemia patterns are identified, then the next step is to make therapy ad- justments to diminish the hypoglycaemia risk and then move on to a summary of the findings and the steps taken to manage these. However it is informative at this point to look at the glucose pattern insights analysis (Figure 5), a traffic light system which accompanies the AGP in the FreeStyle Libre software offered with the FreeStyle Libre flash glucose monitoring system, which flags up the likelihood of low glucose, the proximity of the median glucose to target, and the degree of variability below the median at various times of day (Figure 6). The first two insights identify respectively times at which hypoglycaemia and 
Figure 4. Data over 92 days from a patient using continuous subcutaneous insulin infusion (average total daily dose $43.1 \pm 3.8$ units, $58 \%$ bolus)

\section{a) Raw scattered data}

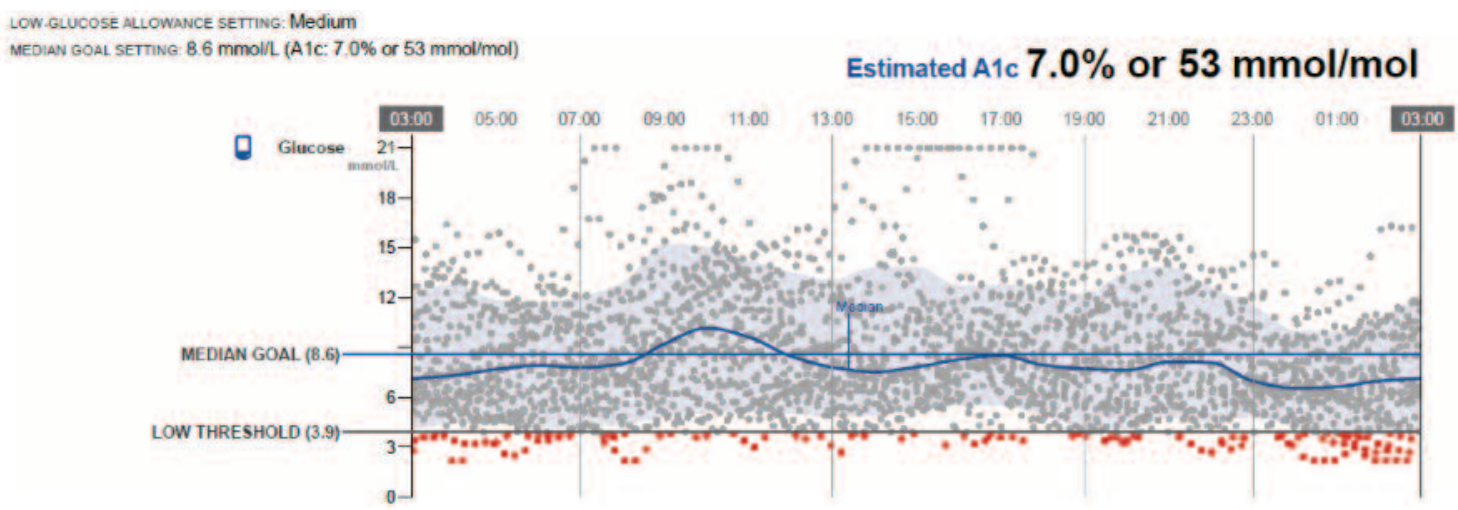

b) The ambulatory glucose profile derived from the same data reveals periods of increased hypoglycaemia risk between 00.00-08.00 and 18.30-20.30.

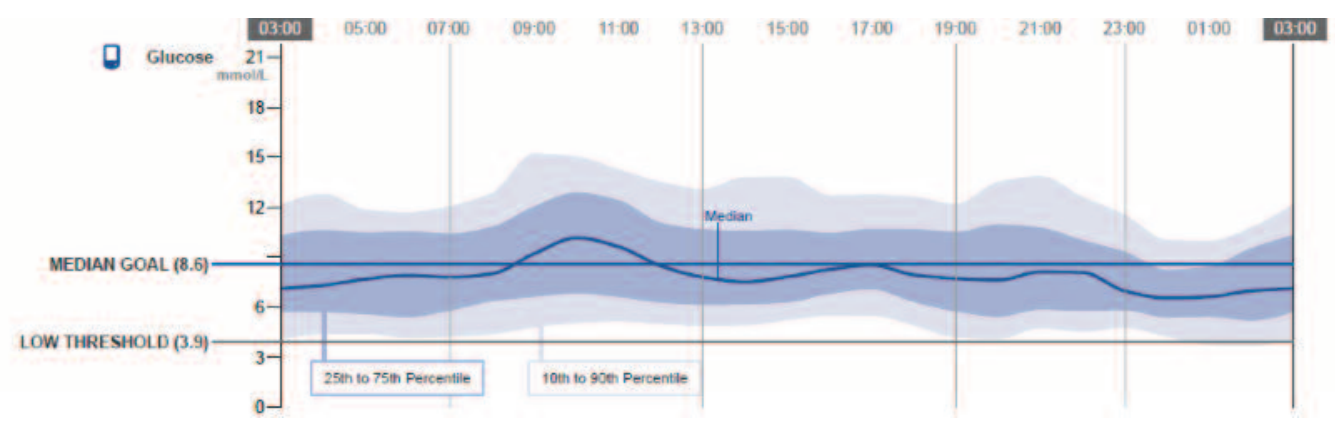

Figure 5. An example of the glucose pattern insights analysis embedded within the software for the ambulatory glucose profile on the FreeStyle Libre flash glucose monitoring system

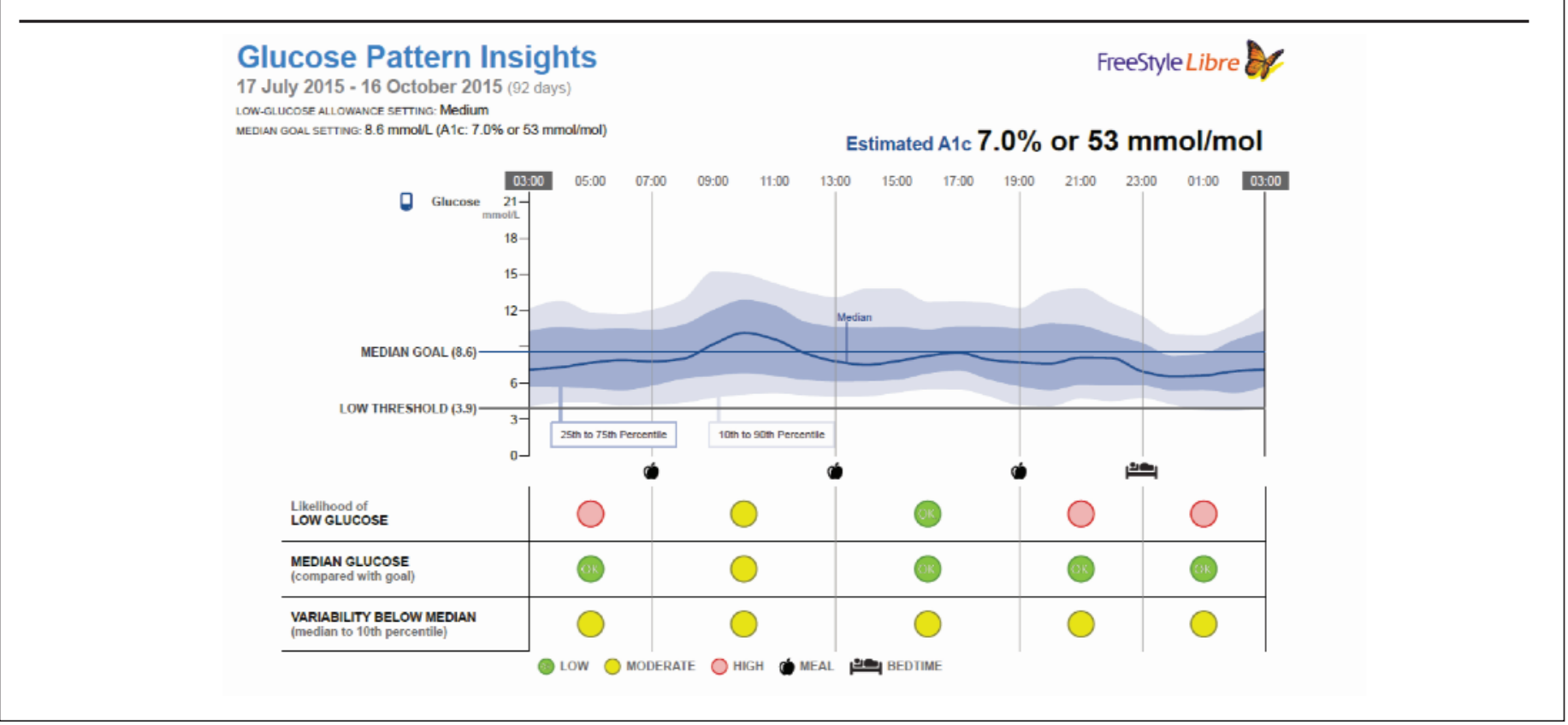


Figure 6. Information on the likelihood of low glucose, the proximity of the median glucose to target, and the degree of variability below the median at various times of day from the glucose pattern insights analysis

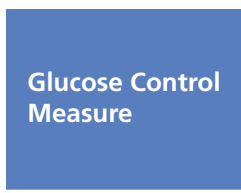

Likelihood of Low Glucose
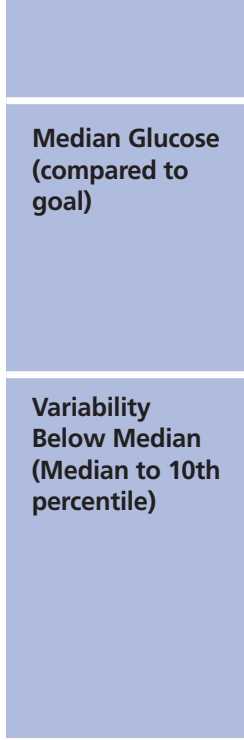

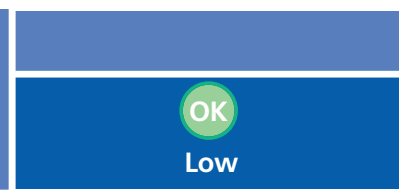

Less than $10 \%$ likelihood of exceeding the low-glucose allowance*

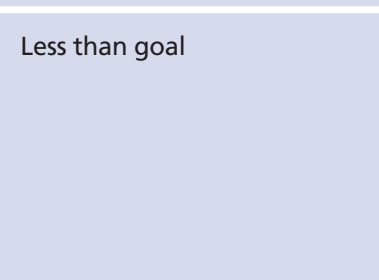

Less than $35 \mathrm{mg} / \mathrm{dL}$ (1.9 $\mathrm{mmol} / \mathrm{L})$

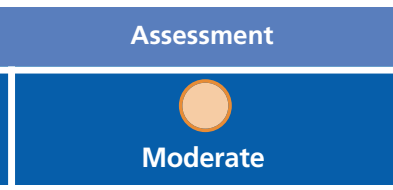

Between $10 \%$ and $50 \%$ likelihood of exceeding the low-glucose allowance*

Greater than goal

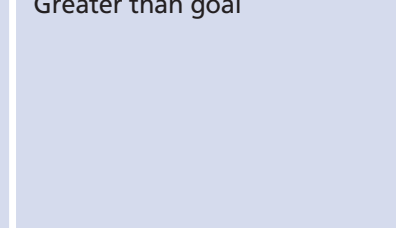

Between Low and High

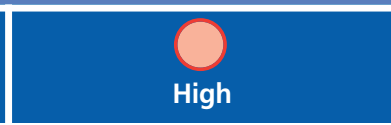

Greater than $50 \%$ likelihood of exceeding the lowglucose allowance*

Greater than goal AND

More than $20 \%$ and $40 \mathrm{mg} / \mathrm{dL}$ ( $2.2 \mathrm{mmol} / \mathrm{L})$ greater than the whole-day median

Greater than a level that would support achieving the Median Goal without potentially causing low glucose

* See Setting Report Parameters section in the FreeStyle Libre software user manual for information about the Low-Glucose Allowance setting

hyperglycaemia risk may be elevated. The third insight may confirm hypoglycaemia risk, but more importantly may identify times where an increase in insulin dosage to address an elevation in glucose levels well above the median might significantly increase hypoglycaemia risk.

Figure 5 allows the initial assessment of hypoglycaemia risk based on the AGP alone to be refined. It is important to note that in this view the $x$ axis is set from 03.00-03.00 rather than 00.00 to 00.00 . As expected both night time periods, 23.0003.00 and 03.00-07.00, are associated with a high (red) risk of low glucose. The period from 19.00-23.00 is also flagged as high risk because the 10th decile line is brushing the low threshold around 19.00-21.00. It is worth noting that in all these time segments the variability below the median is moderate, giving a reasonable degree of confidence about the high risk of low glucose. This confidence would be greater where variability below the median is low as it would imply more consistent risk of low glucose.

A high risk of overnight hypoglycaemia can be addressed immediately as there are unlikely to be any other variables to consider overnight, assuming this is when the user is asleep! A reduction in the basal rate of insulin infusion overnight is the obvious therapeutic adjustment to make.

It is helpful to consider the particular time segment con- cerned in more detail before decisions are made about treatment changes during waking hours. Whilst there appears to be a risk of low glucose between 19.00 and 21.00, there is also increased variability above the median glucose from about 19.30-21.30. Here a review of individual daily profiles and the user's routine is informative. This demonstrates that the risk of low glucose occurs pre-meals and is adequately addressed by the carbohydrate content of the evening meal. Indeed there are occasions where there is a significant peak in glucose values after the evening meal, suggesting that on occasion carbohydrate counting may be inaccurate or the nutrient composition of the meal may be contributing to a delayed peak in glucose levels. Thus no specific therapy changes need to be made to address the AGP profile during this time segment, but the user is now aware that this is an area where more attention is needed and changes may be indicated when further AGP reviews are undertaken.

If hypoglycaemia patterns are not apparent then the consensus guideline recommends assessing each time period in turn: overnight, morning meal, midday meal, evening meal. In the example shown in Figure 5 it is worth noting, before drilling down further into the AGP, that the median glucose is close to target in all segments except 07.00-13.00, and that the estimated $\mathrm{HbA}_{1 \mathrm{c}}$ is close to target at $53 \mathrm{mmol} / \mathrm{mol}$ (7.0\%). Looking at Figure $4 \mathrm{~b}$ again, what observations can we make about each of 


\section{Key messages}

- The ambulatory glucose profile (AGP) contributes to a systematic approach to interpreting dense glucose data from glucose monitoring systems

- Consensus recommendations are available to guide the use of the AGP

- The guidance places emphasis on examination of the AGP for periods of hypoglycaemic risk, i.e. when the 10th centile line for glucose approaches or enters the hypoglycaemic range

- These analyses are supported by the glucose pattern insights analysis available from the FreeStyle Libre flash glucose monitoring system, which flags up the likelihood of low glucose, the proximity of the median glucose to target, and the degree of variability below the median at various times of day

the time periods? We have already addressed the issue of overnight hypoglycaemia so can move on to the other three time periods. There is considerable variability above the median after each meal, with the 90th centile line reaching a similar peak during the midday meal and evening meal segments but a higher peak during the morning meal segment. This is the only time the median crosses above the median goal of $8.6 \mathrm{mmol} / \mathrm{l}$ and the implication is that an increase in the bolus ratio may be needed with the morning meal, a not uncommon requirement.

Again before making any change it is worth reviewing the glucose pattern insights (Figure 5). Note that this is based on 4-6 hour time segments rather than meal-based time periods.
The segment in question is 07.00 to 13.00 where the risk of low glucose is moderate. Therefore we may need to be cautious about increasing the bolus ratio. Looking at the detail, however, it is clear that the 10th decile line is rising from around 08.00 and the risk of low glucose relates to the period from 07.0008.00. The likelihood of low glucose from 13.00-19.00 is low, the only time segment where this is the case, so we can be confident that increasing the bolus ratio with the morning meal will not cause problems with hypoglycaemia in late morning or early afternoon.

\section{Conclusions}

The consensus guideline provides the systematic approach to interpretation which is the cornerstone of using the AGP. A consistent reporting process allows confidence that different members of the diabetes care team, and the user themselves, will reach similar conclusions about the data derived from flash glucose monitoring and CGM and the changes in therapy that are required. A UK consensus guideline will be published shortly which will build on this, and should allow greater access to and understanding of the AGP, and in particular flash glucose monitoring with the FreeStyle Libre system.

Conflict of interest Received honoraria for lecturing and advisory board work for Abbott Diabetes, Novo Nordisk, Lilly, Sanofi, Medtronic, Johnson and Johnson and Roche

Funding This supplement has been supported by a grant from Abbott Diabetes

\section{References}

1. Hammond P. Continuous glucose monitoring: the clinical picture. How to interpret and use the data. Practical Diabetes 2012;29:364-8. http://dx.doi.org/10.1002/pdi.1723

2. Matthaei S, Dealaiz RA, Bose E, et al. Consensus recommendations for the use of Ambulatory Glucose Profile in clinical practice. Br J Diabetes Vasc Dis 2014;14:153-7. http://dx.doi.org/10.15277/bjdvd.2014.046 